\title{
Educación y movimientos sociales. La sostenibilidad de las propuestas
}

\author{
Education and social movements. The \\ sustainability of proposals
}

\author{
Alcira Aguilera Morales ${ }^{1}$ \\ María Isabel González Terreros ${ }^{2}$
}

\section{Resumen}

El presente artículo analiza las convergencias y divergencias en las estrategias que han sostenido a cuatro experiencias de educación lideradas por movimientos sociales de América Latina. En estas se identificaron una serie de aspectos que contribuyen a la permanencia de las experiencias más allá de la financiación. Los aspectos van, desde el establecimiento del vínculo comunitario con el movimiento y las experiencias educativas; la relación en tensión que se encuentra entre las formas de financiación desde la sociedad civil y el estado; los procesos alternativos en la administración y toma de decisiones en los que se desenvuelven las propuestas educativas; la articulación a redes del orden local, nacional e internacional; y la defensa de la autonomía frente a la exigencia de la responsabilidad financiera estatal. Todos ellos son los aspectos que vislumbran tensiones y alternativas en los procesos de sostenibilidad de los proyectos educativos estudiados.

\section{Palabras clave}

Educación, movimientos sociales, Latinoamérica, autogestión, educación alternativa.

\section{Abstract}

This article analyses the convergences and divergences in strategies which have sustained four experiences of education led by social movements in Latin America. In these experiences, we identified certain aspects, beyond financial support, which contribute to the permanency of the experiences. The aspects are as follows: the establishment of the community link with the movement and the educational experiences; the tension between civil society funding and state funding; the alternative processes in the administration and taking of decisions managed by educational proposals; the articulation of local, national and international networks; and, the defence of autonomy faced with the demands of state financial responsibility. All of these aspects indicate tensions and alternatives in sustainability processes of the educational projects studied.

\section{Keywords}

Education, social movements, Latinamerica, self-management, alternative education.

Artículo recibido el 2 de mayo de 2013 y aprobado el 7 de febrero de 2014

1 Docente, Universidad Pedagógica Nacional, Bogotá, Colombia. aamorales@pedagogica.edu.co

2 Docente, Universidad Pedagógica Nacional, Bogotá, Colombia. migonzalez@pedagogica.edu.co 
Universidad Pedagógica Nacional

Facultad de Humanidades

El tema de la educación es central para nosotros porque vemos que si bien los movimientos sociales de América Latina se han constituido en un campo de análisis desde hace varias décadas, de ellos se estudia sus trayectorias y permanencias, el potencial transformador, objetivos e intenciones, banderas de lucha, resistencias y formas de organización, entre otros aspectos necesarios para la reflexión y análisis de los aportes de los movimientos sociales al mundo actual. Pese a ello, el tema de la educación que algunos movimientos de la región gestan, especialmente la sostenibilidad social y financiera de los proyectos educativos, parece estar vedado dado que implica situaciones que pueden considerarse ambivalentes e incluso "incoherentes" con los criterios de los movimientos sociales. En tal sentido, este artículo pretende mostrar las diferentes formas que los movimientos han encontrado para sostener social y financieramente sus propuestas educativas. Para ello estudiamos cuatro casos,

- La Universidad Autónoma Intercultural Indígena (UAIIN), propuesta educativa del Consejo Regional Indígena del Cauca (CRIC) que es la primera organización indígena de Colombia (1971). Su propuesta educativa se viene implementando desde 1978 con escuelas propias, y la UAIIN como resultado del proceso educativo nace en el 2003.

- La Escuela de Nacional de Formación de la Confederación Nacional de Trabajadores Agrarios (ENFOC) de Brasil está articulada a la Confederación Nacional de Trabajadores Agrarios (CONTAG), que agrupa a más de 5 millones de pequeños productores y asalariados del campo brasileño. Junto con el MST, este movimiento es el proceso organizativo más importante en el campo brasileño y con una consolidada experiencia en la formación de sus dirigentes y bases. La ENFOC surge en agosto del 2006.

- La Universidad Intercultural de los Pueblos y las Nacionalidades Indígenas Amawtay Wasi de la Confederación de Nacionalidades Indígenas del Ecuador (CONAIE). Su pro- puesta educativa es reconocida a nivel continental porque propone una educación desde el saber ancestral articulado a la interculturalidad como principio educativo. Experiencia que nace en el año 2004.

- Los bachilleratos populares -Impa y Telar-, que emergen del Movimiento Nacional de Empresas Recuperadas en Argentina en 1998 y que han sido asumidos por otros movimientos populares en Argentina. En la actualidad hay un centenar de bachilleratos populares en la capital del país y la provincia de Buenos Aires.

Como vemos se trata de experiencias educativas que hacen parte de movimientos sociales más amplios y reconocidos a nivel regional: La UAIIN surge del CRIC, la Amawtay se debe a la CONAIE, la ENFOC responde a la CONTAG, los bachilleratos populares hacen parte de la Coordinadora de Bachilleratos articulada a algunos sectores del Movimiento de Fábricas Recuperadas en Argentina. Para estos movimientos, las experiencias educativas son una apuesta política que permite seguir fortaleciendo y potenciando el movimiento social, por ello, las propuestas responden a sus intereses e intencionalidades y han logrado convertirse en proyectos importantes a los cuales los movimientos le dedican tiempos y recursos significativos.

Consideramos que la pregunta por la sostenibilidad nos ayuda a comprender el potencial emancipador, pluralista y contrahegemónico de los movimientos sociales. Se trata de preguntar por el corazón de las experiencias educativas que, en la mayoría de los casos, late como crítica a los modelos tradicionales de educación, al monopolio educativo ejercido por el Estado, a la necesidad de formar sus bases, pero a la vez a una apuesta política por configurar una nueva sociedad. Precisamente, en las cuatro experiencias se manifiesta claramente una crítica al modelo individualista y excluyente que se impone en el mundo, incluso algunas experiencias, como es el caso de los bachilleratos populares - como son denominados por el mismo movimiento-, nacen como respuesta al modelo neoliberal que marginó y 
excluyó a las poblaciones más deprimidas y conllevó a la organización de bachilleratos en la fabricas que quedaron después de la crisis económica que vivió Argentina (2001-2002). Otro movimiento, como la CONTAG plantea la configuración de una escuela que forme a sus cuadros para la comprensión y empoderamiento de su papel dentro del capitalismo y neoliberalismo, así surge la ENFOC, en el año 2006. La CONAIE y el CRIC, proponen desde los años setenta y ochenta educar a sus propias comunidades porque la educación que ofrecía el Estado desconocía la cultura indígena y sus énfasis comunitarios y colectivos, de este proceso surge a inicios de siglo las dos universidades interculturales: la UAIIN en Colombia (2003) y la Amawtay Wasi en Ecuador (2004).

La convicción de que las propuestas educativas son una alternativa para las comunidades que integran el movimiento y que la educación permea los proyectos de vida de los sujetos y sus comunidades, es tal vez, una de las razones más importantes que sostiene a los proyectos educativos, que no en pocas ocasiones se encuentran sin recursos financieros y pese a ello se han logrado mantener por muchos años recurriendo a la autogestión, al trabajo comprometido y voluntario de sus comunidades.

Estas experiencias se sostienen pese a no tener recursos, incluso, pese a que el Estado no reconoce los títulos profesionales que las experiencias otorgan, como es el caso de la UAIIN. Pero ¿Por qué estas propuestas, a pesar de carecer de apoyo financiero, se han mantenido en el tiempo? ¿Qué estrategias han encontrado y construido las experiencias para sostenerse económica y socialmente? ¿Cómo se han sostenido a lo largo de estos años? Estas preguntas no se resuelven con una única respuesta, son tan complejas y ricas las formas de sostenibilidad de las experiencias que interfieren elementos múltiples como estrategias, criterios, redes, apoyos que configuran caminos diversos que cada una recorre. Pero a la vez que existen divergencias también encontramos convergencias en las formas y situaciones a las que han acudido las experiencias para sostenerse en el tiempo.
Para dar cuenta de ello, realizamos un ejercicio comparativo que pretende mostrar la multiplicidad de formas de sostenibilidad que categorizamos en seis componentes que contribuyen a dar continuidad a las propuestas, como se presenta a continuación.

\section{La formación de sus bases: el necesario vínculo comunitario}

Los movimientos sociales que gestan las experiencias educativas tienen vínculos a nivel regional e incluso internacional, lo interesante aquí es mostrar que pese a sus vínculos externos, lo que más contribuye a sostener las experiencias educativas son los vínculos tan estrechos que posee con el contexto local ya que este le da sentido a sus acciones, razón de ser a la propuesta, la defiende y la potencia. En lo local la experiencia educativa encuentra asidero, logra mayor eco y radio de acción tanto para llevar a cabo sus proyectos puntuales, como darle sentido y perspectiva políticopedagógica a las propuestas.

Por ejemplo, los movimientos indígenas que implementan universidades leyeron el contexto de desconocimiento de lo indígena y propusieron una educación que no solo potencie lo étnico, sino que integre diversos conocimientos para favorecer la interculturalidad que se vive en sus territorios y en el país, sin desconocer que dichos movimientos se deben a sus comunidades directas como pasa con las otras experiencias.

Las dos universidades interculturales: la UAIIN y la Amawtay Wasi tienen como vínculo directo y razón de ser a las comunidades indígenas de sus territorios; los bachilleratos populares se deben a los pobladores urbanos de sectores marginados y a los trabajadores; por su parte, la ENFOC integra a los trabajadores rurales del campo brasilero. Aunque, la educación no es exclusiva para estos sectores e incluso en varias de ellas se amplía su radio de acción a la población en general, lo interesante aquí es que se logra establecer un vínculo entre la experiencia educativa y la comunidad local, sean trabajadores, pobladores urbanos, indígenas, jóvenes, mujeres o todos aquellos a quienes los movimientos sociales 
Universidad Pedagógica Nacional

Facultad de Humanidades

acogen y a quienes quiere llegar. El cobijo a los sujetos del orden local es una de las características que más ha contribuido a que los proyectos se mantengan en el tiempo. Por un lado, el vínculo directo con las personas del contexto le otorga soporte y fuerza a nivel local a las experiencias y, por otro, este vínculo que se establece entre el sujeto y el movimiento a partir de la formación educativa hace que las personas se fortalezcan como sujetos y contribuyan a la experiencia, de tal forma que el sujeto se siente reconocido en la experiencia y a la vez la experiencia se siente potenciada por los sujetos que entran a hacer parte de ella.

Precisamente, en las cuatro experiencias educativas estudiadas se establece un vínculo comunitario entre los sujetos y la experiencia que se constituye en "una lucha por el reconocimiento" de sí y el reconocimiento mutuo (Honnet, 1997). Los sujetos que llegan a estas propuestas educativas han hecho parte de procesos de negación, exclusión, minusvaloración o sencillamente de menosprecio, propiciados tanto por el sistema económico que expulsó a los jóvenes del sistema educativo (el caso de los bachilleratos populares), como por las propuestas educativas hegemónicas que desconocen los conocimientos de poblaciones indígenas (las universidades interculturales). De allí que

Los sujetos se encuentran entre sí con expectativas de reconocimiento de las que dependen las condiciones de su integridad psíquica; las acciones colectivas se originan en los sentimientos de injusticia, en la medida en que son experimentados por todo un círculo de sujetos como específicos de su propia situación social. (Honneth, 1997, p. 199)

Estos sentimientos se encuentran en espacios de la vida cotidiana y se anclan a procesos sociales alternativos que se configuran recogiendo a los sujetos que necesitan hacer parte de procesos en donde se potencien, como el caso de los movimientos sociales y especialmente de las experiencias educativas que desarrollan un sistema de educación, profesionalizando a sus actores y por supuesto recogiendo muchas inconformidades por la expulsión y la negación de los sujetos.
De manera que la construcción de esos sentidos de reconocimiento se retoma en las propias experiencias educativas para contribuir en esa lucha de largo alcance contra el menosprecio, la exclusión, la injusticia social en su mayor expresión, sentimientos que se expresan en la construcción de una educación de, para y con los pueblos. Así se distancia radicalmente de la educación (oficial y privada) porque se construye una propuesta educativa que reconoce y acoge a los excluidos por el sistema de educación haciendo de esta una propuesta contrahegemónica. De igual manera, se reconoce la educación de los sectores agrarios y campesinos como contrapropuesta a aquella educación que ha privilegiado los espacios urbanos, tal como se expresa en la experiencia educativa sindical de la ENFOC.

Los movimientos les han otorgado una identidad a los sujetos que configuran sus bases: o son indígenas, campesinos, urbanos, etc. Pese a ello, a las experiencias educativas llegan personas del contexto que se siente o no identificado con los sujetos a los que va dirigida la propuesta. Los contextos locales son plurales en el sentido en que allí habitan pobladores que poseen diversos niveles de compromiso, de identidad, de relación con el territorio, etc. Lo interesante es que a las propuestas también llegan personas que no han tenido vínculos directos con los movimientos y que quieren formarse, ya sea en la universidad, en los bachilleratos o en las escuelas de la ENFOC. Por su parte, las propuestas educativas convocan a las comunidades que se comprometen y hacen parte del movimiento para dar continuidad a los procesos sociales y políticos que llevan a cabo. En el caso de las organizaciones indígenas estudiadas, se acogen principalmente a sus comunidades, aquellas poblaciones que se consideran ancestrales y quienes han venido apoyando los procesos. En el caso de la ENFOC, el proyecto educativo se orienta a la formación de sus líderes sindicales, militantes y bases; $y$, los bachilleratos populares, a los jóvenes y adultos que han sido excluidos de la educación tradicional y que buscan la forma de terminar sus estudios básicos, sin que ello sea una limitante para que lleguen personas del contexto que no responden a los ideales de la organización. 
Esta es la fortaleza de las propuestas educativas porque ubican, en el vínculo comunitario, la potencia para sostener, mantener y dar continuidad a los movimientos sociales que las secundan. En este vínculo se forman las bases para integrarlas a las acciones del movimiento asumiendo procesos de liderazgo. De manera que las experiencias privilegian y propenden porque sus estudiantes sean del territorio y/o de la comunidad, tengan algún tipo de vínculo, en algunos casos que realicen trabajo en ella, o que, con la formación que reciban, se proyecten para ofrecer sus conocimientos a los demás.

Lo anterior se evidencia de dos formas: integrando a las comunidades indígenas a los proyectos educativos, como es el caso de la UAIIN y Amawtay Wasi; o formando a sus delegados, militantes, pobladores y bases para que se oriente la formación en las escuelas como es el caso de la ENFOC y los bachilleratos populares.

En el primer caso encontramos que la UAIIN en Colombia, constantemente está en la búsqueda de que los jóvenes de la comunidad se vinculen a la universidad indígena, especialmente aquellos que hacen parte de los procesos sociales y culturales de la organización. La idea es que los estudiantes asuman responsabilidades sociales como ser maestros comunitarios, agentes de salud, integrantes de la gobernabilidad a través de los cabildos, agentes de la producción y desarrollo ambiental, coordinadores de programas de género, educación, capacitación política, entre otros aspectos (CRIC, 2011). Por su parte y de manera similar, la Amawtay Wasi en Ecuador continuamente se acerca a las comunidades socializando su propuesta para que los comuneros accedan a la formación universitaria, y al mismo tiempo para impedir que la juventud se forme en centros universitarios de la ciudad que desconocen la cultura indígena y que llevan a que el joven no retorne a sus comunidades (Fernando Sarango). ${ }^{3}$

En ambos casos se busca que los profesionales formados en una visión intercultural e indígena se

3 Fernando Sarango coordinador de la Universidad Intercultural Amawtay Wasi. Entrevista realizada por Ma. Isabel González Terreros. Noviembre de 2012. vinculen y aporten a la dinámica organizativa de sus comunidades, lo que supera, en el caso de la UAIIN el problema de la formación superior para buscar empleo (CRIC, 2011) y en el caso de la Amawtay Wasi, que los indígenas se formen en universidades urbanas alejadas de sus conocimientos y realidades. Es decir, no se forma profesionales para que se vinculen a trabajos alejados de sus contextos, por el contrario, uno de los intereses de estas universidades es que apoyen los procesos de las organizaciones sociales y de sus comunidades de manera consciente y comprometida con la intención de dar continuidad a las propuestas.

La relación entre propuesta educativa y formación de las bases proyecta la sostenibilidad de las apuestas políticas y culturales de las organizaciones indígenas. Ello se da porque dicha relación se constituye en el puente entre la política propuesta desde el movimiento y el trabajo que implementa el estudiante y futuro profesional, que contribuye a fortalecer lo comunitario a través de sus apuestas formativas que son de carácter político. En este sentido, la formación "superior" 4 que reciben las comunidades es a la vez el fortalecimiento de un proyecto político y colectivo que se hace más fuerte al apropiar y compartir los sentidos que constituyen el movimiento: mantenerse en comunidad en defensa de sus territorios, la cultura, la lengua, las luchas por el reconocimiento en general.

En el segundo caso, de la ENFOC (2006) y los bachilleratos populares (1998) se plantean la educación de sus bases, líderes y militantes a través de diferentes espacios formativos. En el caso de la Confederación de Trabajadores se tienen escuelas sindicales en las regiones que están en proceso de formación continua de sus militantes y sus bases. ${ }^{5}$ En las escuelas sindicales se fortalece la educación rural para que el trabajador del campo se quede en el campo apoyando los procesos productivos y

4 Entrecomillamos el término, puesto que las propuestas universitarias cuestionan la idea de que existe una educación superior, como si el proceso educativo que le antecede fuese inferior.

5 En la CONTAG, sus bases han estado conformada por líderes sindicales, educadores, líderes de organizaciones eclesiales de base (desde la teología de la liberación), y organizaciones campesinas. 
Universidad Pedagógica Nacional

Facultad de Humanidades

sociales de la CONTAG. A nivel nacional, se realiza un Encuentro Nacional de Formación (ENAFOR) cada cuatro años en el que se reúnen delegados de la confederación y se estimula la apropiación de los principios y fundamentos del Plan Nacional de Formación (PNF) por parte de las escuelas. Aquí hay una intención de las escuelas sindicales por formar a sus propios líderes, militantes, bases y delegados en los principios del movimiento porque ello contribuye a dar continuidad organizativa y política al proyecto. Pero no se trata solamente de formar en política, sino en temas relacionados con la agricultura, el "desarrollo sostenible", el género y el contexto rural, la formación en estos aspectos genera una relación entre la ENFOC y el contexto de los trabajadores rurales quienes en sus comunidades lideran la lucha por la tierra.

Los bachilleratos populares de Argentina también forman a sus jóvenes y adultos, así se fortalece explícitamente la vida comunitaria y la organización (Sverdlick, 2010). Algunos estudiantes, a su vez son trabajadores, padres de familia que fueron excluidos del sistema educativo tradicional. En los bachilleratos, entonces, se pretende no solo integrar a los excluidos, sino que ellos se vinculen a procesos sociales de sus barrios y sectores a partir de la formación que se recibe. En esta propuesta se trasciende la tradicional idea de escuela, haciendo de ella un espacio que integra a los barrios, los pobladores y las organizaciones lo que genera un vínculo de apoyo y fortalecimiento que permite la continuidad de la experiencia en el tiempo. La escuela no está por fuera de lo comunal, en ella los jóvenes y adultos de las fábricas recuperadas y de las asambleas barriales se reúnen para formarse de cara a las necesidades del propio espacio en el que se encuentran (Elisalde, 2011).

En el caso de las universidades es muy claro que se busca integrar a las comunidades indígenas para que ellas ejerzan roles en el movimiento, en el caso de los bachilleratos y la ENFOC se trata de formar a militantes y jóvenes de su contexto para que respondan a las necesidades de sus sectores: rural o barrial, respectivamente. Sin embargo, en todos los casos, la educación no se circunscribe únicamente a la población a la cual se proyecta, pues en todos los casos es claro que su enfoque es abierto y las personas que quieran hacer parte de ello pueden hacerlo.

En síntesis, el vínculo que las experiencias educativas mantienen con los sujetos y organizaciones contribuyen a que las comunidades apoyen decididamente la propuesta, ya sea integrándose a ella y ejerciendo roles dentro del movimiento o fortaleciendo procesos sociales en el contexto que posibilita sumar más apoyos hacia el movimiento. Esto es importante porque las experiencias educativas estudiadas se mantienen y permanecen gracias a la legitimidad que logran en sus espacios territoriales debido a su trabajo de formación y a los proyectos políticos y comunitarios abanderados por los movimientos sociales en los que cada experiencia educativa se inscribe.

Ello implica que hay una especie de "herencia" de proyectos políticos expresada en los principios y los criterios de los movimientos. Es decir, existe una conciencia de delegar liderazgos y responsabilidades a quienes se están formando. Pero unos y otros no es que estén separados, o que unos hagan más parte del movimiento que otros, por el contrario lo que muestran las experiencias es que todos hacen parte del mismo proceso. Por eso los "nuevos" profesionales, bachilleres o líderes rurales no son nuevos para el movimiento sino que se cualifican y se potencian para que contribuyan a mantener la apuesta de los movimientos por la transformación de las sociedades y de su contexto, lo cual reafirma y fortalece la experiencia. De forma que, a través de las propuestas educativas se "heredan" y se mantienen las intenciones, principio y criterios políticos, sociales y culturales del movimiento social.

\section{Entre lo instituido y lo instituyente: dilemas de la financiación}

¿Qué sostiene y cómo se sostienen las propuestas educativas de los movimientos? Si tomamos la pregunta por sí sola, parecería que hace referencia a lo financiero y que se resolvería analizando el origen y uso de los recursos económicos que sostienen a 
los proyectos. Ello se da porque al hablar de la sostenibilidad, la financiación se convierte en un tema central, pese a que existen otros factores que permiten que las propuestas se sostengan, como ya lo hemos venido abordando. Pero, aunque la financiación no es lo central para algunos proyectos, este si es un factor que incide para llevar a cabo proyectos educativos.

En términos generales, frente a la financiación encontramos que los proyectos se debaten en una tensión entre lo instituido y lo instituyente. Es decir, se oscila entre recibir apoyo de organizaciones internacionales que piden cuentas a los movimientos y crear alternativas de financiación autónomas.

Ello se ve muy claro en el caso de la Universidad Intercultural Amawtay Wasi. Cuando esta propuesta nace es sostenida por las comunidades y por sus líderes, igualmente sucedió con la UAIIN. Pero con el tiempo, la Amawtay Wasi fue buscando sostenibilidad financiera con el Estado o con organizaciones internacionales, esta situación la llevó a responder política y administrativamente a dos instancias, la institucional y la comunal:

Las autoridades de esta universidad rinden cuentas a dos instancias a la vez. Por el lado formal estatal, se encuentran sujetas a las regulaciones del Consejo Nacional de Educación Superior (CONESUP). Por el lado no formal o comunitario, las autoridades rinden cuentas a la Confederación de Nacionalidades Indígenas del Ecuador (CONAIE) y al Instituto Científico de Culturas Indígenas (ICCI) [Estas últimas son organizaciones de carácter indígena que sostienen políticamente a la universidad]. (Sarango, 2009, p. 194)

En el caso de la ENFOC, la financiación de las escuelas corre a cargo de la CONTAG que destina recursos del sindicato nacional para la sostenibilidad. Aquí hay una autonomía en la medida en que no responde al Estado sino que son procesos de autogestión. Además la ENFOC no aspira a tener financiación estatal, ni la ha buscado, lo que pretende más bien es lograr independencia económica aceptando el apoyo de otros sectores sociales (nacionales e internacionales) pero con la clara idea de que estas no interfieran en el sentido y desarrollo de la propuesta. La ventaja de las escuelas sindicales es que no hacen parte de la educación formal que conlleva a títulos del sistema de educación estatal, bachilleratos o universidad, sino que su formación es informal, por medio de talleres sistemáticos para mantener la lucha por la tierra/el campo, aprender acerca de procesos agrícolas autónomos y que respeten el medio ambiente.

Por su parte la UAIIN no tiene solvencia económica, pero posee varias formas de financiación. Una es el apoyo de la comunidad y otra es la gestión ante instancias nacionales e internacionales. Por un lado, los estudiantes apoyan económicamente con una cuota de $\$ 500000$ por ciclo, que en algunos casos es solventada por los cabildos como instancias que proponen a líderes para que sean formados profesionalmente. Ello se debe a que en un Congreso del cric se determinó que los cabildos debían "destinar el $2 \%$ de los recursos de SGP [Sistema General de Participación] para los estudiantes que vienen acá a la UAIIN" (Carlos Juagiboy). ${ }^{6}$ La otra forma de financiación es el apoyo internacional que se traduce en recursos económicos para la producción de materiales escritos, educativos, audiovisuales y la consolidación de la UAIIN (Bolaños, 2008), o de apoyo de instituciones de educación superior que contribuyen a viabilizar procesos de formalización y titulación porque la UAIIN no es una institución reconocida por el Ministerio de Educación de Colombia.

En el caso de los bachilleratos populares, especialmente algunos que se encuentran en la ciudad de Buenos Aires, han logrado que el Estado destine recursos para el pago salarial de docentes y becas estudiantiles (Roberto Elisalde). ${ }^{7}$ En esta lucha por tener financiamiento estatal se reivindica que la responsabilidad de la educación es del Estado argentino, a través de los recursos públicos. En esa medida se rechaza la idea de acudir a la financiación privada y se exige la financiación por parte del Estado como

6 Orientador (docente) de la UAIIN. Entrevista realizada por Alcira Aguilera Morales a Carlos Juagiboy, abril 2012.

7 Docente y gestor de los bachilleratos populares. Intervención realizada en el marco del Encuentro educación en movimientos sociales, UPN. Noviembre de 2012. 
Universidad Pedagógica Nacional

Facultad de Humanidades

un derecho de los ciudadanos. Se trata de defender un derecho que a su vez resignifica el sentido de lo público, pues buscar financiación estatal no contraviene el sentido de la educación pública como acto político de liberación y transformación social (Finnegan, 2009). Pese a ello, existen algunos bachilleratos que no aceptan financiación por parte del Estado porque consideran que si se paga salario a los maestros ellos ya no responderían al movimiento y a su compromiso social y político, sino que deben responder a las políticas educativas nacionales, desvirtuando el horizonte.

La forma en que se sostienen financieramente las propuestas van desde una autonomía total como es el caso de la ENFOC que es apoyada económicamente por el movimiento (CONTAG) a una relación tensa de apoyo formal y organizativo como es el caso de la Amawtay y en cierta forma de los bachilleratos que definen cada uno si recibe o no apoyo del Estado. Esta relación allá y acá, que parecería ambivalente, es la apuesta de las organizaciones y de sus proyectos educativos por mantenerse. Se trata de una apuesta política entre lo instituido (el Estado y las ONG) y lo instituyente (las organizaciones sociales y comunitarias). Esta jugada a "dos bandas" que se usa en tres de los cuatro casos, les posibilita a las propuestas educativas tener relaciones con dos "enemigos históricos", las organizaciones sociales y el Estado.

De acuerdo con estos procesos de financiación, aludiremos a la noción de imaginario de Castoriadis (citado en Poirier, 2006) para ver su potencia en relación con los procesos instituyentes de financiación y autonomía que se gestan en las experiencias estudiadas. En primer lugar lo imaginario se refiere a aquello que se está creando permanentemente, que no es reflejo de algo dado de antemano y que por tanto se da como indeterminado en términos históricos, sociales e incluso psíquicos (Castoridis, citado en Poirier, 2006). Al retomar el proceso de financiación como "imaginario", se evidencia que las experiencias educativas están en un proceso de sostenibilidad que las ha mantenido y les dado continuidad por medio de alternativas de autogestión, mediadas por tensiones entre lo instituido e instituyente. Se trata de un imaginario liminal ya que estas alternativas se desarrollan en medio de la incesante tensión por crear nuevas formas de financiación, autogestionarias, en respuesta en muchos casos, a la negativa estatal de reconocerlas y por tanto de apoyarlas económicamente.

De esta manera, hablar de imaginario en el ámbito de la sostenibilidad financiera permite ver las creaciones e invenciones con las cuales las propuestas educativas asumen el reto de autofinanciarse. Este imaginario se encuentra en las propuestas educativas de los movimientos sociales estudiados, ya que estas surgen como opciones alternativas, en el sentido de buscar crear nuevas formas de sostenibilidad para promover una educación que responda y se articule a los movimientos sociales.

Lo imaginario desde la creación permanente se ve en el hecho de que las experiencias no nacen con un carácter oficial-estatal, ni privado, sino comunitario o popular organizado como dicen los bachilleratos. Ello ha implicado que no se consideren "privadas" por el hecho de cobrar matrícula, pues estas no representan cobros altos y, por el contrario, se buscan formas de sostener financieramente a los estudiantes y en general a la experiencia. Del mismo modo, el hecho de no considerarse oficial estatal, en el sentido que no se basan en la educación hegemónica que plantea el Estado, lleva a que el Estado no las financie ni destine recursos para su sostenibilidad, por lo que ellas deben recrear formas de sostenerse por medio de la autogestión de recursos. Se trata de un proceso instituyente que apela tanto a la capacidad de gestionar recursos, desde abajo con alternativas que se dan a nivel local, regional o internacional; como de mantener la autonomía política y social con la que se construye la experiencia.

Así, lo que las sostiene es una continua creación de estrategias, alianzas y formas para conseguir la sostenibilidad de las experiencias educativas que hacen parte del movimiento. Por ejemplo, la Universidad Intercultural de las Nacionalidades y Pueblos Indígenas Amawtay Wasi nace en el 2004 como una "institución particular autofinanciada" 
(Sarango, 2009) siendo el mayor de los retos para su sostenimiento económico el cobro de pensiones a un costo mínimo, de forma que se logra mantener el equipo técnico y académico de la institución sin que se limite el acceso a un mayor número de estudiantes de escasos recursos. Así, la Amawtay Wasi, se ha proyectado como una propuesta autosostenible, que recurre a los siguientes elementos para garantizar su sostenimiento económico:

Propenderá en el más breve lapso posible a que el funcionamiento económico y financiero sea autosostenible; - Los apoyos económicos que se obtengan serán en lo posible para financiar las investigaciones, emprendimientos y becas de los estudiantes; - Se realizará un manejo lo más trasparente de los recursos, considerando la realización de auditorías y evaluaciones externas periódicas; - Se realizará un manejo económico financiero global, por centros del saber, por ámbitos de formación, considerando las comunidades de aprendizaje, los cuis, entre otros aspectos. (CONAIE, 2004, p. 244)

De igual manera ocurre con la UAIIN del CRIC, la cual nunca ha contado con el apoyo financiero por parte del Estado ni del reconocimiento oficial, en su lugar ha acudido a financiación por parte de organizaciones nacionales e internacionales, por ejemplo, del Banco Interamericano de Desarrollo, del mismo Consejo Regional Indígena del Cauca y, por supuesto, de la comunidad.

En el caso de la CONTAG (Brasil), con su Escuela Nacional de Formación Sindical se viene creando el Fondo Solidario que aporta el $1 \%$ de las mensualidades de los pensionados para la formación. Sin embargo, esto sigue siendo insuficiente para lograr la autonomía financiera de la escuela. Esta experiencia sirve como reflexión sobre la necesidad y la posibilidad de una alternativa financiera independiente y estratégica para la escuela siendo un caso poco común en el mundo sindical brasileño (CONTAG, PNF, 2006, p. 46). La necesidad de construir un proyecto con total autonomía financiera responde a un principio fundamental en el movimiento y es la defensa a su capacidad de autodeterminación, la cual puede ser vulnerada por los diferentes agentes finan- ciadores, quienes pueden intervenir en las decisiones, principios y orientaciones político-pedagógicas.

Por último, con respecto a los bachilleratos populares (Impa y Telar), se encuentra que estas propuestas educativas hacen parte de organizaciones sociales sin ánimo de lucro. Al no contar con una base financiera establecida de manera fija por el Estado han acudido a diversas fuentes de financiación para garantizar el funcionamiento de la propuesta, entre ellas encontramos: el cobro de matrículas a los estudiantes, donaciones de fundaciones sin ánimo de lucro, subsidios extraordinarios ofrecidos por el Estado, el aporte solidario de docentes e investigadores de la Universidad de Buenos Aires y del apoyo de la comunidad de vecinos de los barrios en que se ubican los bachilleratos (Elisalde, 2011, pp. 53-55). La segunda fuente de financiación se encuentra en las organizaciones nacionales e internacionales que posibilitan la consecución de recursos para sostener y mantener en funcionamiento las propuestas educativas. Por último, y de manera marginal, se menciona el apoyo económico estatal a través de la figura de subsidios extraordinarios, como ocurre en la experiencia de los bachilleratos populares (Impa-Telar).

En síntesis, encontramos algunos imaginarios en las formas de financiación de las propuestas educativas surgidas de, con y para los movimientos sociales en América Latina: La principal ha sido la autosostenibilidad, la autogestión de recursos que no comprometan las políticas de la experiencia educativa. Estas son características en las que convergen las convicciones políticas de los diferentes movimientos, que encuentran como primer elemento para sostener sus propuestas el autofinanciamiento. La autosostenibilidad se expresa en la consecución de recursos financieros a partir de sus propias organizaciones de base (CRIC, CONAIE, CONTAG, etc), del cobro de matrículas a sus estudiantes y el apoyo de las comunidades locales que creen en estas apuestas educativo-políticas, así como de las solidaridades internacionales. 
Universidad Pedagógica Nacional

Facultad de Humanidades

\section{La movilidad en las formas administrativas}

Definir la forma como se organizan a nivel administrativo las diferentes propuestas educativas es muy complicado debido a la diversidad de estructuras y momentos en que se da su funcionamiento. Es decir, la estructura de las propuestas educativas es de diversa índole y no se puede enmarcar en una forma particular, porque aunque algunas propuestas tengan una estructura similar a la vertical: órganos nacionales, regionales que inciden en la experiencia educativa, o una estructura denominada pirámide inversa en la que se encuentra la dirección en la parte más baja de la pirámide porque ella responde a toda la base social; o a pesar de que otras experiencia se ubiquen con estructuras horizontales donde todos hacen parte del todo y no hay jerarquías tan verticales, lo cierto es que no siempre se funciona de la misma forma, y estas formas se complejizan en la práctica.

Las estructuras no son totalmente horizontales, verticales o piramidales, más bien se trata de formas complejas en donde múltiples tipos de estructura se trasponen e integran, para tomar decisiones por ejemplo, o en donde los sujetos ocupan diferentes espacios-lugares en la estructura administrativa. Preferimos tomar como ejemplo una especie de "telaraña" para poder entender la complejidad administrativa en las experiencias educativas. Por una parte, se evidencia que todos los componentes: consejos, direcciones, coordinaciones y sujetos se entrecruzan y de alguna manera están conectados entre sí. Pero, por otra parte, no se trata de ocupar un espacio determinado y rígido dentro de la estructura sino que los sujetos se mueven de lugar.

Veamos los ejemplos. Los bachilleratos populares hacen parte de la Coordinadora de Bachilleratos Populares (CBP), un espacio de organización en el cual confluyen organizaciones sociales, territoriales y políticas de diverso tipo. Pero, a la vez, cada uno de estos bachilleratos tienen autonomía sobre sus proyectos educativos, e incluso se diferencian de acuerdo a si reciben apoyo del Estado para sus maestros, o los que deciden no recibir sueldo. De tal forma que aunque hacen parte de la Coordinadora de Bachilleratos cada uno asume su rumbo, lo que muestra que no se trata ni de una estructura vertical en donde la Coordinadora está arriba y es el poder central sobre el cual todos los bachilleratos se asumen, pero tampoco una propuesta horizontal porque hace parte de una RED de bachilleratos que confluye en la opción por los excluidos del sistema educativo.

Otro ejemplo lo podemos encontrar en la UAIIN. La universidad responde directamente al CRIC. Es decir, el centro de la telaraña estaría representado por el CRIC, que es el máximo organismo del movimiento. Si el CRIC está en el centro, en el término medio estaría la UAIIN, que a su vez debe responderle a toda la comunidad que estaría en la parte más externa de la telaraña. Aquí, con una mirada simplista podríamos decir que existen unas jerarquías definidas. Sin embargo, la comunidad que está en el lado amplio de la telaraña es la que decide sobre quienes pueden ser apoyados para estudiar, hasta hacen parte de los procesos de evaluación para definir qué estudiantes serán promovidos o sobre qué profesiones requiere la comunidad para que la UAIIN las viabilice. El centro, el medio y la parte externa de la telaraña, entonces, no se pueden ver como una pirámide, sino desde las múltiples conexiones que se establecen allí.

Estos ejemplos los tomamos solamente para ver la figura de la telaraña, porque ella nos permite mostrar que existe una movilidad en la administración de cada una de las propuestas educativas de los movimientos sociales, en el sentido en que no solo se mira de arriba hacia abajo, del centro hacia afuera, de un lado al otro y viceversa, sino que se asumen formas administrativas y de decisión complejas. Existe una relación hacia fuera con el movimiento, pero las decisiones no siempre son supeditadas al movimiento sino a las mismas poblaciones a las que se deben. Veamos cada uno de los casos de manera más específica.

Como decíamos anteriormente, la UAIIN responde al Consejo Regional Indígena (CRIC), que está conformando por nueve consejeros que hacen parte de cada una de las zonas indígenas del Cauca. 
El CRIC es la máxima instancia del movimiento y tiene tres coordinaciones: educativa, administrativa y política. Cada una de ellas es acompañada por dos o tres consejeros y a su vez por un coordinador que no es consejero. Al componente del Sistema Educativo Indígena Propio (SEIP), pertenece la UAIIN que es, a su vez, coordinada por una persona que tiene relación con la estructura antes mencionada y dentro de la UAIIN existen coordinadores por cada programa que se ofrece. La estructura administrativa de la UAIIN, en particular,

Cuenta con varios órganos de decisión: El Consejo Político Cultural, encargado de orientar y dirigir las decisiones generales que atañen al funcionamiento político de la UAIIN. ${ }^{8}$ [...] A su vez se encuentra el Consejo Académico, encargado de revisar, orientar y viabilizar la creación, funcionamiento y seguimiento evaluativo a cada programa. ${ }^{9}$ [... E El Consejo Administrativo, tiene como función el orientar la organización operativa, la consecución de recursos para que la UAIIN pueda cumplir con cada una de sus líneas de acción. ${ }^{10}$ [...] La Coordinación General integrada por el delegado de cada uno de los consejos en el ánimo de adelantar una coordinación colegida. (Bolaños, 2008; Bolaños, Tattay, y Pancho, 2009)

Ahora bien, en términos de decisiones la situación se complejiza, no es la anterior estructura la que toma decisiones, sino que estas son discutidas, decididas desde abajo y en asambleas comunales. La estructura administrativa es la encargada de ejecutar las decisiones, orientarlas, evaluarlas. Son las comunidades quienes en primera instancia

8 Este consejo está conformado por las respectivas autoridades políticas de los territorios indígenas que hacen parte de la UAIIN, entre ellos: Consejería Regional, Coordinación PEBI, Asociación de Cabildos, Cabildos, Alcaldías indígenas o comprometidas con el proceso, La Organización Nacional Indígena de Colombia (ONIC), la Coordinación Política de la UAIIN. También forma parte de este consejo las autoridades espirituales y mayores, quienes tienen la responsabilidad del acompañamiento y orientación espiritual en la toma de decisiones

9 Este consejo está conformado por la Coordinación del PEBI, la Coordinación de cada uno de los programas, la Coordinación Pedagógica de la UAIIN, los cabildos de estudiantes, principalmente.

10 La Coordinación Administrativa de la UAlıN y la Coordinación Administrativa del PEBI, conforman dicho consejo. Además se encuentra dentro de sus órganos deciden en asamblea de acuerdo a sus necesidades, preguntas, problemas sociales e intereses políticos. Por ejemplo, en los resguardos se evidencia que no existen formas claras para aplicar la justicia propia, por ello plantean que se requiere de personas que ayuden a pensar y "aplicar" el derecho propio desde la identidad de las comunidades. Se propone formar comuneros para que estudien el tema y en el futuro contribuyan a abordar el asunto. La decisión de la asamblea entonces es que la universidad se encargue de ello. Así el CRIC, como máxima instancia de representación debe tratar este tema, y junto con la UAIIN, los orientadores, líderes, cabildantes, coordinadores, autoridades espirituales comienzan a pensar el asunto para dar vida al programa de Justicia Propia o Ley Natural.

La universidad ecuatoriana está constituida por órganos colegiados como el Consejo Universitario, Consejo Ejecutivo, los Centros del Saber. ${ }^{11}$ "Las autoridades principales son: Rector, Vicerrectores Académico de Investigación y Administrativo Financiero, Coordinadores de los Centros del Saber y el Secretario General - Procurador que conforman el Centro del Saber Kawsay" (Sarango, 2009, p. 194) Como vemos, no aparece la CONAIE dentro de la estructura administrativa, a pesar de la relación estrecha que existe entre la universidad y el movimiento. Aquí se plantea una autonomía frente a la organización, pero sin desconocerla.

Se podría decir que es una estructura tradicional como la que cobija a todas las universidades. Pero en términos de la organización y la forma en que en la Amawtay se toman decisiones, ellos afirman que se parte de la idea de una pirámide trunca "que proyecta el sentido de una organización de abajo hacia arriba, donde la sumatoria organizada de colectivos pequeños constituye un colectivo grande llamado universidad" (Sarango, 2009, p. 194). Se podría entender entonces que en la parte superior es donde se encuentran todas las personas y en general las comunidades que hacen parte de Amawtay, y que

11 Los centros del saber son: Kawsay o de la Vida; Ushay Yachay o de la Interculturalidad; Ruray Ushay o de las Tecnociencias para la vida; Munay Ruray o del Mundo Vivo; y Yachay Munay o de las Cosmovisiones. 
Universidad Pedagógica Nacional

Facultad de Humanidades

en la parte de abajo están los consejos y las autoridades principales. De forma que, las decisiones son asumidas de abajo hacia arriba para que todos participen en ellas.

La Escuela Nacional de Formación Sindical, funciona internamente con un equipo de Coordinación Política desde donde se coordina lo pedagógico, los convenios, los proyectos y la producción de materiales. Además cuenta con un Consejo Político Gestor que es un órgano colegiado ampliado responsable de la interlocución y articulación nacional en la dirección política de la Escuela. (CONTAG, PNF, 2006)

Lo que se decide a estos niveles es ejecutado por el equipo operativo que está integrado por la "Asesoría de Formación" y la Secretaría de Formación además de la red de colaboradores con quienes se ejecutan las tareas de las coordinaciones mencionadas. Existe, a su vez, una Secretaría Administrativa que organiza la escuela y es responsable de los contactos internos y externos y de manejar las bases de datos de los integrantes de la Escuela.

Aquí se muestra una verticalidad de la estructura, tal vez porque la ENFOC pertenece a un sindicato, y es bien sabido que los sindicatos se han consolidado con formas rígidas. Pese a ello, la estructura les ha permitido mantenerse, porque a pesar de ser una escuela nacional de un país tan grande y diverso como Brasil, la escuela ha logrado su consolidación. Es la forma operativa más acorde para coordinar las escuelas sindicales a nivel nacional, siendo un reflejo del centralismo democrático que asumen muchas organizaciones sindicales.

En el caso de los bachilleratos populares del CEIP (Colectivo de Educación e Investigación Popular) el trabajo desde la Coordinadora de Bachilleratos Populares (CBP) se configura como un espacio de organización en el que confluyen organizaciones sociales, territoriales y políticas con carácter de independencia y autonomía frente al Estado y los gobiernos. Si nos detenemos a mirar a cada una de las organizaciones que hacen parte de la Coordinadora vamos a ver la misma variedad o tal vez más de la que hemos encontrado en estas cuatro experiencias. Los bachilleratos se articulan con organizaciones, no solo educativas sino territoriales, sindicales y políticas, pero cada bachillerato asume formas interesantes de administrarse, casi siempre en relación con organizaciones políticas de los maestros que lideran los bachilleratos. Aquí habría múltiples centros de la telaraña y varias fugas e interconexiones que se articulan y se rompen.

Todas ellas son formas administrativas que interpelan en gran medida la idea de una administración centralizada, con órganos de ejecución decisional únicos, en su lugar se encuentran formas complejas de administración y cada una ha venido implementando su estructura desde sus propias construcciones. Se observa que existe una diversidad y complejidad de formas de administrar y estructurar las propuestas educativas. En términos muy generales, lo que las experiencias comparten es la idea de organismos de dirección; participación de las poblaciones por medio de congresos o asambleas; la toma de decisiones como un ejercicio de todos los sujetos, no solo de la dirección; y la diversidad de roles que cumple el sujeto del movimiento.

\section{Las decisiones y participación como corresponsabilidad}

En primer lugar, las experiencias comparten la idea de organismos de dirección encargados de viabilizar las decisiones o demandas tanto de las poblaciones como de los organismos que lideran los movimientos. Para ello, las experiencias han configurado consejos o coordinaciones. Tres de las cuatro organizaciones tienen organismos de dirección denominados consejos: "el Consejo Universitario y el Consejo Ejecutivo" (Amawtay); "el Consejo Administrativo, el Consejo Académico" (CRIC); "el Consejo Político Gestor" (ENFOC), ninguno de los consejos se denomina igual, pero se refieren a instancias colegiadas en las que participan representantes de la organización, de la experiencia educativa y en algunos casos de las poblaciones (Amawtay Wasi, UAIIN), para administrar algún asunto clave de la propuesta educativa: lo académico, lo político, lo administrativo, o todas al tiempo, etc. 
Ahora bien, en cuanto a la idea de coordinación, en los bachilleraros populares aparece esta figura de manera central, lo mismo que en la ENFOC, y aunque en el CRIC también está la idea, ella se refiere más a un cargo administrativo y de gestión no a una figura central de la coordinación, es decir que no tiene la misma centralidad que en las demás organizaciones. Ello muestra una diferencia, los movimientos indígenas prefieren hablar de consejos y los bachilleratos populares y la ENFOC de coordinación, aunque aparentemente se refieren a lo mismo: instancias de representación del movimiento y de deliberación.

En segundo lugar, los consejos y las coordinaciones son organismos políticos y administrativos que centralizan las decisiones que se toman desde las asambleas o congresos. En la mayoría de las organizaciones se dan espacios de asambleas (CRIC, Amawtay, bachilleratos) o congresos (CRIC, Amawtay Wasi y ENFOC). Las asambleas son reuniones cotidianas en las que se reúne la comunidad para discutir y, en algunos casos, decidir asuntos que consideran claves para el devenir de las mismas y de los movimientos. Por su parte, los congresos son menos cotidianos, se realizan cada cierto tiempo: un año, dos o más y se toman decisiones sobre el rumbo a mediano y largo plazo. Lo que ambos espacios comparten es que allí participan una gran cantidad de personas afines a las propuestas educativas y también a los movimientos sociales que las sustentan.

La participación es un tema y una forma de hacer que las experiencias comparten. En todos los casos se muestra la necesidad de que sus comunidades participen tanto en la ejecución de sus acciones como en la validación de las decisiones. En todas ellas existe una idea de participación, sea porque, en el caso de la ENFOC, los delegados regionales llevan las iniciativas de las bases a instancias encargadas de tomar decisiones, o porque en amplias asambleas las comunidades, que hacen parte de las organizaciones como el CRIC, participan expresando sus puntos de vista. Esta necesidad de tener en cuenta la participación de diferentes actores del movimiento social es fundamental para dar continuidad o "avalar" las propuestas que se ejecutaran.

Consideramos que la relación entre toma de decisiones y participación nos da pistas para entender por qué las experiencias educativas de estos movimientos sociales se mantienen en el tiempo. Los sujetos de los movimientos participan de la toma de decisiones en diferentes momentos, por ejemplo cuando consideran hacer un cambio o viabilizar algunas propuesta, ellos plantean en asamblea o reunión el asunto y este es tratado. Allí participan, pero también cuando debe ser ejecutado o evaluado el proyecto, las personas apoyan y dan su punto de vista. Es decir, el hacer parte de la toma de decisiones no denota que el sujeto se circunscribe a un solo momento o a una sola "sección" del movimiento y de la decisión, sino que interviene a lo largo del proceso. Aquí hay una mirada diferente de la participación, no se trata de delegar la decisión, sino que se asume hasta dar viabilidad a lo planteado, así se generan mecanismos de corresponsabilidad, reconocimiento del proceso, interiorización de la acción y mayor involucramiento en la experiencia.

El hecho de que los diferentes actores sociales de las organizaciones tengan interiorizado el mapa en el que participan para asumir, proponer, aprobar, ejecutar y evaluar las decisiones, es una potencia para la sostenibilidad porque los hace sentir parte de un todo, de un engranaje que ha sido pensado por muchos y en el cual el sujeto en términos particulares tiene la posibilidad de hacer parte.

Este elemento nos lleva a plantear que los sujetos que participan en las experiencias se mueven de arriba hacia abajo, y de un lado para el otro, dependiendo del proceso de las acciones del movimiento. Es decir que no existe un espacio fijo en donde el sujeto se ubique sino que cambia de rol, lo que le permite tener un mapa más general del movimiento y desarrollar múltiples capacidades al ejecutar diferentes tareas.

Es interesante ver que a pesar de que las propuestas de educación tienen organismos políticos $\mathrm{y}$ administrativos que centralizan las decisiones que se toman desde las asambleas o congresos (CRIC, 
Universidad Pedagógica Nacional

Facultad de Humanidades

CONAIE, MSTTR, CEIP), estos no solo asumen el mandato de las poblaciones, sino que buscan la forma de viabilizarlas porque quienes conforman la dirección del movimiento social a la vez hacen parte de las poblaciones, por lo tanto suben y bajan de la "pirámide", o en el mejor de los casos, se mueven de un lado al otro en la telaraña, van del centro al borde para que las decisiones sean ejecutadas. Ello le permite al coordinador desplazarse de un lado a otro o de arriba hacia abajo cuando va a la asamblea de los pobladores, o cuando debe hacer parte de las acciones para ejecutar las decisiones. De manera similar lo hace un estudiante o una persona de la comunidad porque decide en asamblea pero realiza acciones para llevar a cabo las decisiones

Aunque existen roles, estos no son tan marcados, y sobre todo tan limitantes, como en las institucionales formales. Podemos decir que existen diferentes instancias dentro del movimiento que proponen, avalan, ejecutan y evalúan las decisiones. Ello no es diferente a otras organizaciones institucionales, pero lo alternativo y realmente instituyente es que todas las instancias participan en diferentes momentos de los procesos de decisiones. Es decir, en la mayoría de organizaciones existen las poblaciones a las que va dirigida la propuesta educativa (las bases), existen órganos de coordinación de la propuesta: consejos, coordinaciones, pero a la vez órganos de direccionamiento de los movimientos sociales: las comunidades, los pobladores, los sindicalistas, los trabajadores del campo. Estas tres instancias participan en los diferentes momentos del proceso: proponer, avalar, ejecutar, evaluar, etc.

Se trata de que los sujetos sientan que hacen parte de todo el proceso, que son parte de la dirección y coordinación del proyecto, como de la base que ejecuta y decide. Aquí, a pesar que hay una labor específica que cada sujeto cumple dentro del proceso (estudiante, maestro, coordinador, trabajador, líder), se busca que este también haga parte del todo. Es decir que si es estudiante también ejerza de líder, de trabajador, de maestro, de cabildante y de coordinador. Por ejemplo, en la UAIIN, un coordinador del programa de pedagogía a la vez participa del órgano de dirección de la universidad, también va a las comunidades para dar cuenta de sus procesos y tomar decisiones que no solo atañen a la pedagogía comunitaria o a la UAIIN en general, sino al movimiento social. Este estar en varios momentos del todo, el estar en varios espacios del todo, el responder a tareas diversas, hace que la experiencia se mantenga porque logra establecer lazos afectivos y comunales más amplios, y más allá de las relaciones escolares tradicionales (maestro-estudiante).

La forma en que se asumen las decisiones en las organizaciones que tienen a cargo las propuestas educativas también da pistas sobre qué las sostiene en el tiempo. Es decir, por qué a pesar de que algunas experiencias no tienen presupuestos del Estado e incluso no son reconocidas oficialmente, siguen funcionando. Consideramos que una de las pistas la podemos encontrar en la forma como se toman las decisiones. Por una parte hay una gran participación en ellas, pero por otra, las organizaciones tienen formas complejas por medio de las cuales los sujetos que hacen parte de la propuesta no solo participan en diferentes momentos sino que conocen el mapa de la organización y las instancias por las que pasa una decisión, en las que se combinan procesos decisorios de carácter horizontal y/o vertical, sin privilegiar el centralismo democrático (democracia representativa) o la total horizontalidad (democracia directa).

Este hacer parte del todo a partir de unos roles especiales pero no limitantes, y a la vez el tener el mapa del todo y saber cómo se toman, avalan, ejecutan y evalúan las decisiones, permite a los sujetos del movimiento sentirse parte central del mismo e involucrarse afectivamente en él. En este aspecto se teje una nueva visión del ejercicio político que permite pensar a largo plazo los sueños colectivos inscritos en los movimientos y en sus apuestas educativas.

\section{La integración a redes hermanas y amigas}

Las organizaciones sociales y en particular sus propuestas educativas encuentran en las redes regionales y en la solidaridad de personas particulares un apoyo importante para seguir con sus procesos. La 
intención es articular solidaridades en torno a los proyectos que llevan a cabo cada una de las organizaciones que hacen parte de la red con el objetivo de fortalecer mutuamente las propuestas sin comprometer la autonomía que se tiene. Es decir, se realizan procesos de articulación en red o coordinaciones con otros, o se reciben solidaridades de personas o instituciones para apoyos académicos o financieros siempre y cuando ello no comprometa los principios, los objetivos y en general la autonomía de las experiencias educativas.

Frente a la integración en red encontramos que las experiencias tratan de establecer relaciones con organizaciones que comparten perspectivas políticas, sociales y educativas. Esto se evidencia en las cuatro organizaciones. Los bachilleratos populares establecen relaciones de hermandad y solidaridad con los otros bachilleratos y con experiencias de fábricas recuperadas a nivel nacional. En la ENFOC se trata de un trabajo de acompañamiento de académicos que apoyan la experiencia; mientras que en el caso de las dos universidades interculturales ambas se encuentran haciendo parte de la misma red y de otras redes de América Latina que se configuran para apoyarse en procesos educativos y proponer alternativas frente a los procesos de acreditación, entre otros intereses que detallaremos posteriormente.

La ENFOC recibe apoyo de académicos que prestan asesorías a la experiencia. Para la organización ellos "son una red conformada por especialistas que tienen afinidad política y pedagógica, que hacen de consultores con una mirada externa sobre las formas de hacer educativas, desde las áreas de las cuales son especialistas" (CONTAG, 2006, p. 49).

En el caso de las universidades interculturales, estas se articulan a procesos de formación indígena para fortalecer sus proyectos educativos, interculturales y organizativos, sobre todo con experiencias de América Latina. La Amawtay Wasi, por ejemplo, hace parte de la Red Tinku que vincula iniciativas indígenas en Perú y en Bolivia. Tinku trata de unificar iniciativas para el pueblo quechua o quichua en la perspectiva de construir la gran Confederación
Quechua que está difuminada por Bolivia, parte de Argentina, Chile, Perú, Ecuador y Colombia. Además dicha red posee una relación con universidades de Dinamarca, Finlandia y Suecia. La UAIIN también ha establecido vínculos y hace parte de la Red de Universidades Indígenas de la cual participan experiencias de Ecuador, Nicaragua, México y Bolivia. La idea de esta red es establecer acuerdos y políticas de las universidades de educación superior intercultural para el fortalecimiento de la sabiduría y la cosmovisión indígena con enfoque intercultural y propio.

La UAIIN y la Amawtay Wasi han establecido vínculos con universidades indígenas de Latinoamérica a partir de encuentros, convenios y apoyos. Un ejemplo de ello fue el Segundo Encuentro Internacional de Universidades Indígenas adelantado durante el 5 y 6 de agosto de 2009 en la ciudad de Quito, Ecuador. En él participaron varias universidades interculturales de América Latina (Ecuador, Nicaragua, México, Bolivia). Pero no se trató solo de encontrarse, que ya es un avance, sino de establecer acuerdos y políticas de fortalecimiento de la Red de Universidades Indígenas. Por ello se acordó trabajar en el fortalecimiento de la Red de Universidades Indígenas, Afrodescendientes e Interculturales que permitan el fortalecimiento de la educación superior, la sabiduría y la cosmovisión indígena con enfoque intra e intercultural; promover acciones conjuntas para fortalecer los espacios y los conocimientos ancestrales e implementarlos en los planes de estudio, mallas curriculares de las universidades, para tener una educación propia y unificada entre las universidades; conformar equipos de gestión y búsqueda de recursos para garantizar la sustentabilidad de las universidades interculturales y el fortalecimiento de la red; realizar actividades de intercambio, investigaciones, pasantías, así como proyectos conjuntos entre las universidades, para fortalecer los procesos académicos de formación, acreditaciones y techos académicos propios.

El dialogar con otras experiencias, establecer intereses comunes, realizar plataformas de lucha, y proponer trabajo conjuntos a mediano plazo, 
Universidad Pedagógica Nacional

Facultad de Humanidades

conlleva a que los movimientos y sus experiencias educativas no se vean ni se sientan solos, nadando contra la corriente, y por el contrario, sientan que existen otras experiencias que están andado por caminos similares. Son propuestas contrahegemónicas frente a los actuales procesos de evaluación y acreditación, ya que lo claro es que estas propuestas tienen su propio valor y especificidad y no tendrían por qué medirse con los mismos criterios que las universidades oficiales.

En el caso de los bachilleratos populares, estos hacen parte del Colectivo de Educación e Investigación Popular (CEIP), que a su vez hace parte de la misma red de asociación y están nucleados en la coordinadora. Es decir, su red está integrada por múltiples bachilleratos populares $-\mathrm{y}$ sus organizaciones- que surgen en Buenos Aires y a nivel nacional y se articulan a la Coordinadora de Bachilleratos Populares en Lucha, la cual agrupa a la mayoría de las experiencias. Lo cierto es que existen otras organizaciones gremiales con perspectivas orientadas a fines distintos, tales como La Red y Batalla educativa.

En cuanto a los apoyos particulares, las organizaciones sociales y en particular sus propuestas educativas, buscan apoyos en instituciones o personas solidarias y comprometidas e instituciones públicas o privadas. Algunos apoyos son puntuales frente a una acción o proyecto, en otros casos se trata de apoyos sostenidos en el tiempo, varios años incluso. Ello ha llevado a que algunas experiencias establezcan tipo de apoyos, por ejemplo en la ENFOC se diferencian tres tipos de relación con otros actores sociales o institucionales: colaboraciones, cuando son puntuales en torno a un evento o acción; parcerias, cuando son permanentes y asociadas a un proceso o a un campo de acción o a temática estratégica; y alianzas, cuando se establecen vínculos estables con organizaciones con identidad política e ideológica.

Esta división tan particular de los apoyos no corresponde a todas las propuestas aunque todas los reciben. En el caso de la UAIIN recibe apoyos de instituciones nacionales como la Universidad del Cauca, la Universidad Pedagógica Nacional, con quienes se tiene convenios de cooperación, y también la Universidad Nacional, Universidad de Antioquia, Universidad de Los Andes, Universidad San Buenaventura, entre otras. Por su parte la Amawtay Wasi recibe el apoyo de académicos interesados en la propuesta, más que apoyos de instituciones como tal, se trata de apoyos de algunas personas que hacen parte de las instituciones. En el caso de los bachilleratos los apoyos están más en las organizaciones gremiales como la Unión de Trabajadores de la Educación (UTE), la cual representa a todo docente que se desempeñe en dicho campo en la ciudad de Buenos Aires, independientemente que sea en el sector público o privado.

Los vínculos que establecen las organizaciones sociales con otras experiencias o con otros sujetos, lo mismo que el trabajo en red, es un soporte político importante porque se basa en los vínculos políticos y comunitarios a nivel nacional y regional que permite asumir los procesos de forma más colectiva y mancomunada. Pero la idea es que este apoyo o articulación no comprometa la autonomía para seguir configurando propuestas alternativas y contrahegemónicas.

\section{Autonomía y Estado: la relación en permanente tensión}

Las experiencias educativas aquí abordadas presentan tensiones y disputas con el Estado, en unos casos más que en otros, pero en todos hay un nivel de tensión entre movimiento y estado por algunas situación de la educación. Es decir, que aunque el hecho de ser movimiento social que lucha por algunos derechos o reivindicaciones sociales ya distancia a la organización del Estado, aquí vamos a abordar el cuestionamiento central: ¿Cuál de las propuestas educativas alternativas que agencian estos movimientos está en disputa con el Estado?

La financiación es uno de aquellos elementos. Por un lado, dos experiencias (UAIIN y la Amawta Wasy) luchan frente al Estado no solo por el reconocimiento como institución de educación sino también por financiación porque sus comunidades tienen derecho a la educación y el Estado debe pro- 
veer los recursos. Por otra parte, se encentra otra experiencia (ENFOC) que no le solicita recursos al estado porque ellos se autogestionan y no están dispuestos a perder su autonomía. Finalmente, en lo referente a los bachilleratos, algunos están entre la lucha por la financiación de maestros y otros decidieron no asumir esa lucha para no perder su autonomía.

Pese a las diferencias en cuanto a recibir, luchar o disputar recursos o reconocimiento por parte del Estado, el hecho de que en todas las experiencias se acuda a otras formas de financiación que confrontan el desconocimiento por parte del Estado es un elemento que reivindica la capacidad de autonomía y autogestión dentro de las experiencias educativas. Precisamente, esta lucha por la autodeterminación y la autonomía se expresa de diferentes maneras en cada una de las experiencias educativas.

Es el caso de la ENFOC, que reivindica la autonomía total por lo que no disputa recursos del Estado, pero sí se recibe ayuda externa. No obstante, en caso de obtener apoyos económicos, no se permite a terceros incidir en sus propuestas y decisiones. Los bachilleratos populares, por su parte, se dividen esta disputa, ya que algunos reclaman un Estado garante de la educación como derecho pero que no interfiera en la selección y construcción del proyecto educativo, popular y público; otros bachilleratos sencillamente no aceptan ningún apoyo económico estatal. La Amawtay Wasi, aunque recibe apoyos económicos del Estado, reclama autonomía en las decisiones y en la construcción de la propuesta políticoeducativa; caso similar al de la UAIIN, a la que se le reconoce autonomía total, pero sin dejar de lado la exigencia de un Estado responsable financieramente.

Sin embargo, especialmente en el caso de las dos universidades interculturales una de las diputas más fuertes se da en torno a la lucha por el reconocimiento y la autonomía ante sus respectivos Estados, ya que se oponen a ser evaluadas y acreditadas con los mismos parámetros de cualquier universidad tradicional. Esta posición nace de su condición de autonomía, que podemos entender como la capacidad de transformar las propias instituciones sociales, así como el derecho a transformarlas (Castoriadis, citado en Poirier, 2006). Desde esta concepción ambas universidades reclaman la financiación estatal, financiación que ya fue obtenida en parte por la Amawtay Wasi. Las universidades interculturales reivindican la autonomía para decidir sobre sus procesos de formación, evaluación, seguimiento, currículo etc., no quieren someterse a modelos de medición y acreditación externas y globales porque ellos están diseñados para la homogenización cultural y no para que los movimientos indígenas se revindiquen como comunidad intercultural. La acreditación es una forma de medición que desconoce los sentidos, razones de ser y los procesos instituyentes que se gestan en ambas experiencias.

Estos procesos tensionan la vieja idea de autonomía educativa, pues si bien reivindican el apoyo estatal, lo hacen reclamando total autonomía frente al mismo. En las experiencias educativas se reconoce que los apoyos financieros estatales no se pueden entender como un sinónimo de intromisión. Este aspecto se puede ilustrar acudiendo a otros sectores de la sociedad, tal es el caso de la salud o los tribunales de justicia, al entenderse que son instituciones financiadas por el Estado y no por ello puede ser violentada su autonomía en torno a la toma de decisiones, la administración de la justicia, entre otros aspectos fundamentales para orientar los destinos de un país. Como lo menciona Boaventura De Sousa Santos (2006), al referirse a la defensa de la autonomía universitaria acudiendo a una analogía con el sistema judicial, "en el que la independencia de los tribunales no se afecta por el hecho de que sean financiados por el Estado" (p.24).

De acuerdo con este análisis en las cuatro experiencias educativas es evidente que se asume la autonomía como capacidad de transformar las propias instituciones sociales, así como de decidir el tipo de educación y de vida que se quiere vivir, esta es la autonomía que está en juego en las experiencias educativas.

Estas tensiones solo reafirman que los movimientos sociales están tomando la educación en sus manos, y vienen instituyendo nuevas formas de su 
Universidad Pedagógica Nacional

Facultad de Humanidades

quehacer educativo, así como de la construcción de autonomía. Esta concepción de autonomía reclama para sí misma la no interferencia del Estado ni de terceros como la Iglesia o los intereses del mercado. De forma que los movimientos vienen instituyendo un proceso de concepción de lo educativo que confronta las ideas de universidad, educación y escuela, concebidas por el proyecto moderno, que cuestionan la educación bancaria en sentido freireano o para el ascenso social. Los movimientos generan alternativas al monopolio educativo que ejerce el Estado, tanto en el sector público como en el privado, al recuperar el sentido de la educación para la transformación de los pueblos.

Vemos así, que las experiencias educativas en mención se constituyen en alternativas al confrontar las visiones educativas tradicionales y capitalistas $y$, por tanto, constituirse en propuestas que recrean lo comunitario. Tal como lo expresa Santos Boaventura (2003), en América Latina asistimos a la expresión de un paradigma tránsito o transición paradigmática, en el que cobra vital importancia la construcción de una vida digna y decente a través de la recuperación y reinvención del principio de la comunidad. Es en este aspecto en el que se evidencia la potencia y existencia de las experiencias educativas, pues la comunidad se entiende como solidaridad, participación y vínculos en los que cada miembro se siente reconocido e incluido, vínculos en los que se trasciende el individualismo y los valores capitalistas, vínculos que motivan a dar vida y defender ese proyecto de educación alternativa y digna para los pueblos excluidos y marginados.

\section{Referencias}

Ampudia, M. (2011). Movimientos sociales, sujetos y territorios de la educación popular en la Argentina de la década del 2000. En Ampudia M, et alt, Trabajadores y educación en la Argentina. De las estrategias sindicales a la acción de los movimientos sociales. Buenos Aires: Buenos Libros.

Bolaños, G. (Mayo-agosto, 2005). Ustedes y nosotros, diferentes mas no inferiores... La construcción de un proyecto indígena en Colombia. Revista Educación y Pedagogía, 19(48).
Bolaños, G. (2008). Universidad Autónoma, Indígena e Intercultural: Un espacio para el posicionamiento de Epistemologías diversas (Colombia). En D. Mato (Coord.), Diversidad cultural e interculturalidad en educación superior. Experiencias en América Latina. Caracas: Instituto Nacional para la Educación Superior en América Latina y el Caribe (IESALC), Unesco.

Bolaños, G., Tattay, L., Pancho, A. (2009). Universidad Autónoma Indígena e Intercultural (UAIIN): Un proceso para fortalecer la educación propia y comunitaria en el marco de la interculturalidad [Colombia]. En D. Mato (Coord.), Instituciones Interculturales de Educación Superior en América Latina. Procesos de construcción, logros, innovaciones y desafíos (pp. 155199). Caracas: Instituto Nacional para la Educación Superior en América Latina y el Caribe (IESALC), Unesco.

Confederación de Nacionalidades Indígenas del Ecuador (CONAIE) (2004). Proyecto político de la conaie, declaración política. Consultado el 7 de marzo de 2012. Disponible en http://www.conaie.org/ sobre-nosotros/que-es-la-conaie

Confederación de Nacionalidades Indígenas del Ecuador (CONTAG) (2006). Plan Nacional de Formación (PNF). Brasil: CONTAG-ENFOC.

Consejo Regional Indígena del Cauca (CRIC) (2004). ¿Qué pasaría si la escuela...? 30 años de construcción de una educación propia. Bogotá: Ed. El Fuego Azul. Bogotá.

Consejo Regional Indígena del Cauca (CRIC) (2009). Caminando la palabra de los congresos del CRIC. Febrero de 1974 a marzo de 2009. Popayán: PEBI/ UAIIN/ Gobierno Vasco/CRIC.

Consejo Regional Indígena del Cauca (CRIC) (2011). Sistema educativo indígena propio. SEIP. Primer documento de trabajo. Sin ciudad, CRIC

Consejo Regional Indígena del Cauca (CRIC) (2012). La UAIIN. Consultado el 7 de marzo de 2012. Disponible en http://cric-colombia.org

Elisalde, R, Ampudia M, J. P. Nardulli, y J. Calvagno, (2011) Trabajadores y educación en la Argentina. De las estrategias sindicales a la acción de los movimientos sociales. Buenos Aires: Buenos Libros.

Fernández, A. (2008). Política y subjetividad: asambleas barriales y fábricas recuperadas. Buenos Aires: Biblos.

Finnegan, F. (2009). Educación popular y educación de jóvenes y adultos: algunas reflexiones sobre un diá- 
logo complejo entre tradiciones diversas. Paraguay: IDIE Mercosur.

González, M, I. (Primer semestre de 2013). Las convergencias educativas de los movimientos sociales en América Latina. Transitando otros caminos. Ideacão, Revista do Centro de Educacão e Letras Campus Foz de Iguaçu, 15.

Honneth, A. (1997). La lucha por el reconocimiento. Por una gramática moral de los conflictos sociales. Barcelona: Ed. Crítica.

Poirier, N. (2006). La imaginación radical. En C. Castoriadis, Imaginario radical. Buenos Aires: Nueva Visión.

Salazar, E. (2012). Análisis de la experiencia de la Universidad Intercultural de las Nacionalidades y Pueblos Indígenas del Ecuador, Amawtay Wasi (Casa de la sabiduría). En L. Buitrago et ál., Análisis de experiencias educativas en organizaciones y movimientos sociales en América Latina: Estudio de caso [Tesis de Grado]. Maestría en Educación. Pontificia Universidad Javeriana.

Santos, Boaventura de Sousa (2006). La universidad del siglo Xx. Para una reforma democrática y emancipadora de la universidad. Cuba: Ed. Casa de las Américas.

Santos, Boaventura de Sousa (2003). Critica de la razón indolente. Contra el desprecio de la experiencia [Volumen I]. Madrid. España: Descleé de Brouwer.

Sarango, L. (2009). Universidad Intercultural de las Nacionalidades y Pueblos Indígenas "Amawtay Wasi”. Ecuador/Chinchaysuyu. En D. Mato (coord.), Instituciones Interculturales de Educación Superior en América Latina. Procesos de construcción, logros, innovaciones y desafíos. Caracas: IESALC-Unesco.

Sverdlick, I. (2010). Experiencias educativas que nos interpelan. El sentido del derecho a la educación, el formato escolar y las políticas públicas. Consultado en http://www.rizoma-freireano.org/index.php/ experiencias-educativas-que-nos-interpelan-elsentido-del-derecho-a-la-educacion-el-formatoescolar-y-las-politicas-publicas

Universidad Intercultural de las Nacionalidades y Pueblos indígenas Amawtay Wasi (2004). Sumak Yachaypi, Alli Kawsaypipash Yachakuna. Aprender en la sabiduría y el buen vivir. Learning Wisdom and the Good to Live. Quito: UNESCO.

Universidad Intercultural de las Nacionalidades y Pueblos indígenas Amawtay Wasi, (Junio de 2008).
Educación y movimientos sociales. La sostenibilidad de las propuestas Alcira Aguilera Morales, María Isabel González Terreros

La experiencia de la universidad Intercultural de las Nacionalidades y Pueblos Indígenas “amawtaywasi”. Hacia un Nuevo Paradigma de Educación Superior. Revista Yachaykuna 9.

Universidad Intercultural de las Nacionalidades y Pueblos Indígenas Amawtay Wasi, (2008). Universidad Autónoma, Indígena e Intercultural: Un espacio para el posicionamiento de Epistemologías diversas (Colombia). En D. Mato (coord.), Diversidad cultural e interculturalidad en educación superior. Experiencias en América Latina. Caracas: Instituto Nacional para la Educación Superior en América Latina y el Caribe (IESALC), Unesco. 